\title{
Entwicklung eines angepassten Vorgehensmodells für Data-Warehouse-Projekte bei der W\&W AG
}

\author{
Gertrud Heck-Weinhart, Gabriele Mutterer
}

W\&W Informatik

\section{Clemens Herrmann, Josef Rupprecht}

Universität St. Gallen

Vorgehensmodelle im Software Engineering werden zur Strukturierung des Entwicklungsprozesses sowie zur Reduktion der Komplexität herangezogen. In diesem Artikel wird die Entwicklung eines an die $W \& W$-spezifischen Verhältnisse angepassten Vorgehensmodells für Data-Warehouse-Projekte erläutert. Als Basis dienen die Erfahrungen dreier, teilweise abgeschlossener Data-Warehouse-Projekte, eine Schwächenanalyse vorhandener und angewandter Vorgehensmodelle sowie Ergänzungen aus der Literatur. Das entwickelte Soll-Vorgehensmodell für die $W \& W A G$ berücksichtigt darüber hinaus die speziellen Rahmenbedingungen und Vorgaben des Konzerns.

\section{$1 \quad$ Einleitung}

Vorgehensmodelle zur Informations- und Anwendungssystementwicklung sind in der Praxis weit verbreitet und akzeptiert. Der Einsatz von Vorgehensmodellen im Rahmen des Software Engineering ${ }^{1}$ hat zahlreiche Vorteile (vgl. o. V. 1997, S. 756):

- Explizite und eindeutige Strukturierung des Entwicklungsprozesses,

- Komplexitätsreduktion in Entwicklungsprojekten durch eine Spezifikation von Teilproblemen,

- Verdeutlichung von Zusammenhängen und Abhängigkeiten zwischen Aufgaben und Phasen,

- Auflistung aller relevanten und durchzuführenden Aufgaben und

1 Für eine Übersicht über Vorgehensmodelle aus dem Software Engineering vgl. (Bremer 1998; Balzert 1998, S. 97-133). 
- Personenunabhängigkeit der Anleitungen und Hilfestellungen für das Entwicklungsprojekt.

Data-Warehouse-Projekte sollten ebenfalls durch Vorgehensmodelle unterstützt werden können. Allerdings sind in Unternehmen angewandte Vorgehensmodelle mehrheitlich an den Belangen der Entwicklung operativer Systeme ausgerichtet. So existierte auch im W\&W-Konzern bisher kein detailliert dokumentiertes Vorgehensmodell speziell für die Entwicklung von Data Warehouses. Das vorhandene Vorgehensmodell für IT-Projekte im Allgemeinen musste daher von jedem Projektleiter und jeder Projektleiterin auf die besonderen Belange des jeweiligen Projektes angepasst werden. Dies war zeitaufwendig und gelang unterschiedlich gut abhängig vom Erfahrungswissen der Projektbeteiligten.

Um dieses spezielle Wissen für künftige Data-Warehouse-Projekte unabhängig von dem Projektleitenden verfügbar zu machen, sollte ein idealtypisches Vorgehensmodell für Data-Warehouse-Projekte entwickelt werden. Aus diesem Grund sollte personengebundenes Erfahrungswissen expliziert und über eine Stärken- und Schwächenanalyse Good-Practices identifiziert werden. Hierzu wurden bereits abgeschlossene und in Gang befindliche Entwicklungsprojekte untersucht und dokumentiert. Angereichert mit Literaturergänzungen wurde daraus ein spezifisches Vorgehensmodell für Data-Warehouse-Entwicklungsprojekte bei der W\&W AG erarbeitet.

Ziel dieses Artikels ist die Darstellung der Beweggründe und Schritte zur Entwicklung dieses idealtypischen Data-Warehouse-Vorgehensmodells für die W\&W Informatik.

In Abschnitt 2 wird ein allgemeiner Überblick über Vorgehensmodelle im Data Warehousing gegeben. In Abschnitt 3 erfolgt zuerst eine kurze Darstellung der drei Data-Warehouse-Projekte bei der W\&W Informatik, die den Ausgangspunkt für das Soll-Vorgehensmodell bilden. Daraus werden die Spezifika von Data-WarehouseProjekten abgeleitet, die in dem Vorgehensmodell berücksichtigt werden sollen. Die zu beachtenden Rahmenbedingungen der W\&W Informatik werden ebenfalls skizziert. Im Abschnitt 4 wird das entwickelte Data-Warehouse-Vorgehensmodell erläutert und aufgezeigt, welche Verbesserungen im Projektablauf gegenüber dem bisherigen Vorgehen erwartet werden können. Der Artikel schliesst mit einer Zusammenfassung und einem Ausblick auf den noch vorhandenen Handlungsbedarf. 


\section{Vorgehensmodelle im Data Warehousing}

Die Entwicklung von Data-Warehouse-Systemen unterscheidet sich vom Entwurfsprozess für operative Informationssysteme teilweise erheblich. Während operative Anwendungssysteme Geschäftsprozesse, Abläufe und Funktionen unterstützen bzw. umsetzen, steht bei einem Data Warehouse die Bereitstellung einer konsistenten, integrierten und historisierten Datenbasis zur Entscheidungsunterstützung im Vordergrund. Dieses Charakteristikum der starken Datenorientierung muss auch im Vorgehensmodell zur Entwicklung eines Data Warehouse zur Geltung kommen. Insbesondere in den Anfangsphasen eines Data-Warehouse-Projekts treten im Vergleich zur traditionellen Applikationsentwicklung bedeutende Unterschiede auf.

Weitere spezielle Anforderungen an ein Data-Warehouse-Vorgehensmodell resultiert aus der typischerweise mehrere Verantwortungsbereiche übergreifenden Querschnittsfunktionalität eines Data Warehouse (vgl. Simon 1998, S. 6).

Aus beiden Charakteristika begründen sich die zusätzlich durchzuführenden Aktivitäten aus dem Bereich der Datenbankentwicklung (z. B. Vossen 1994, S. 48 ff.; Heuer, Saake 2000, S. 171 ff.), die stärkere Betonung des Projektmanagementaspekts sowie die folgenden kritischen Erfolgsfaktoren für Data-Warehouse-Entwicklungsprojekte (vgl. Poe et al. 1997, S. 74-81; Jung, Winter 2000, S. 18):

- Eine exakte Definition der zu erreichenden Ziele aufgrund von Problemen in den Fachbereichen und eine Darstellung der Nutzenpotenziale im Rahmen des Projektmarketings ist notwendig.

- Data-Warehouse-Entwicklungsprojekte sollten nach angemessener Zeit nutzbare Resultate gemäss des „Think big - start small“ Ansatzes liefern. Iterative Vorgehensweisen sind zu präferieren, da i. d. R. zum Projektbeginn eine vollständige Spezifikation der Anforderungen nicht möglich ist.

- Lösungen müssen in enger fachlicher Zusammenarbeit mit den Endbenutzern erarbeitet werden. Die Einbindung der Endbenutzer in den Entwicklungsprozess ist erforderlich für eine benutzergerechte und zielgerichtete Lösung und damit für die Akzeptanz von Data-Warehouse-Systemen.

In der Literatur finden sich einige theoretische Vorschläge für Vorgehensmodelle auf unterschiedlichsten Abstraktionsniveaus für den Aufbau von Data-WarehouseSystemen, welche die oben beschriebenen Eigenheiten teilweise berücksichtigen (z. B. Holthuis 1999, S. 220-233; Meyer, Strauch 2000; S. 97 ff., Hansen 1997; Kachur 2000). 


\section{Data-Warehouse-Projekte bei der W\&W}

Für die Entwicklung des Data-Warehouse-Vorgehensmodells der W\&W Informatik waren die Erfahrungen in den im Folgenden dargestellten drei Projekten massgeblich. Die Beschreibung der Projekte konzentriert sich auf das Vorgehen im Projekt und die für das Data-Warehouse-Vorgehensmodell relevanten Erkenntnisse.

\subsection{W\&W Kundendatenbank - WWK-Projekt}

\subsubsection{Inhalt des Projektes}

Durch die Fusion von Wüstenrot Gruppe und Württembergischer Versicherungsgruppe und der anschliessenden Verschmelzung der Leonberger Bausparkasse auf die Wüstenrot Bausparkasse mussten sich insbesondere die Bereiche Vertrieb und Marketing völlig neuen Herausforderungen stellen. Mit dem Projekt einer dispositiven W\&W Kundendatenbank (kurz WWK) sollte eine konzernweite Lösung zur Unterstützung von Marketing und Vertrieb im Cross-Selling geschaffen werden. Das WWK-Projekt stellte das erste konzernweite Data-Warehouse-Projekt der W\&W Informatik dar.

In einer 1. Stufe des WWK-Projektes sollte eine konsistente, integrierte Datenbasis bereitgestellt werden. Wichtiger als die Vollständigkeit der Daten waren deren Konsistenz und die Berücksichtigung des gesamten Konzern-Kundenbestandes. Auch wurde die schnelle Bereitstellung erster Übersichten der vollständigen Implementierung aller wünschenswerten Funktionalitäten vorgezogen.

Mittelfristig sollten die Datenbasis und danach die darauf aufbauenden Anwendungen in mehreren Stufen zu einer konzernweiten Vertriebs- und Marketingdatenbank mit kompletter Funktionalität - Bestandsmarketing, Cross- und Up-Selling, Database Marketing usw. - ausgebaut werden.

\subsubsection{Verwendetes Vorgehensmodell}

Das für WWK Stufe 1 verwendete Phasenmodell orientiert sich an den Vorgehensmodellen für IT-Projekte innerhalb der W\&W Informatik. Die übergeordneten Schritte zur Etablierung der konzernweiten Kundendatenbank sind in Abb. 1 dargestellt.

Dieses Vorgehensmodell beinhaltet folgende allgemein gültigen Regeln: Für jedes Projekt werden jeweils alle Phasen durchlaufen. Innerhalb eines Projektes sind die Phasen partiell iterativ. Mögliche bzw. sinnvolle Rückschritte in vorangegangene Phasen sind möglich und eingezeichnet. Die „Studie“ stellt in diesem Phasenmodell ein optionales, eigenständiges Projekt dar, welches durchgeführt wird, um die Projektrisiken detaillierter zu evaluieren bzw. unklare Sachverhalte genauer zu untersuchen. Grundsätzlich wird nach jeder der konzeptionellen Phase entschieden, ob das Projekt fortgesetzt oder gestoppt wird. 


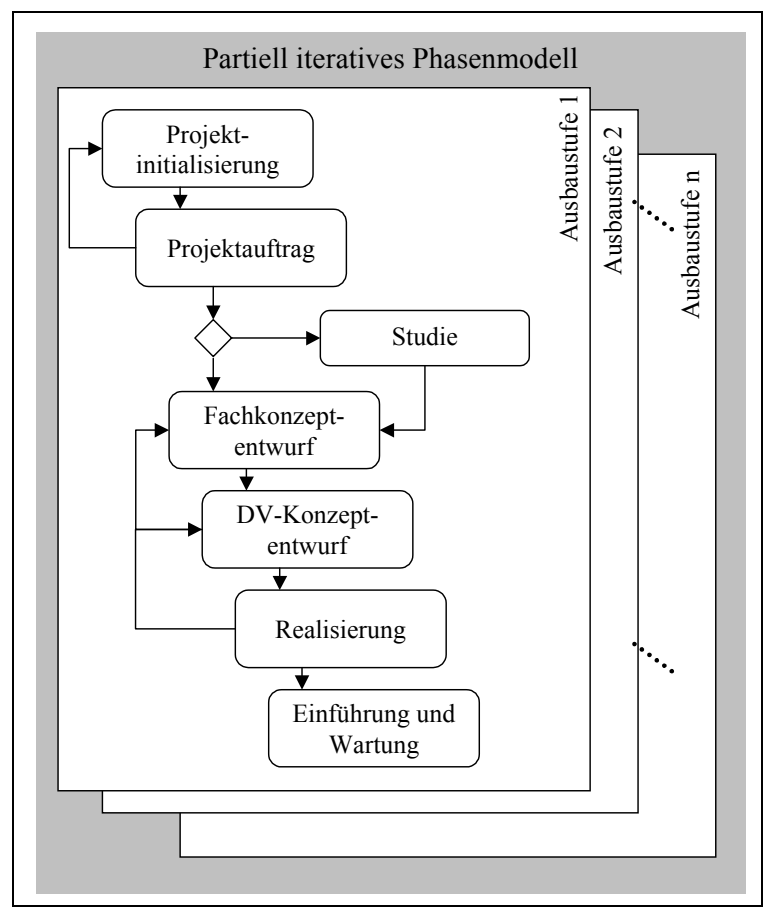

Abb. 1: Phasen des WWK-Projekts

\subsubsection{WWK-spezifisches Vorgehen für Stufe 1}

Die ersten Überlegungen zu dem vorgestellten Projekt wurden in der Projektinitialisierung von der Fachabteilung angestrengt, allerdings hatte noch kein Entscheid über die tatsächliche Durchführung stattgefunden. Diese Projektanfrage wurde von der IT-Abteilung in Form eines Projektangebots beantwortet, welches einen ersten abstrakten Entwurf eines Lösungsvorschlags enthielt. Ziel des anschliessenden Projektauftrags war es, die Projektausgestaltung genauer zu spezifizieren und Kriterien bzw. Rahmenbedingungen zu ermitteln.

Anforderungen aus Sicht der Nutzer waren insbesondere die Verfügbarkeit von benötigten Daten aus allen Konzernbereichen sowie von Standardabfragen und -selektionen, periodischen Berichten und Analysen. Aus Datensicht ergaben sich fachliche Anforderungen in erster Linie an die Vereinheitlichung der Begrifflichkeiten, da dieselben Begriffe in jedem Konzernteil eine andere Bedeutung hatten und noch haben. Technische Anforderungen betrafen Aktualität, Qualität und Historienführung der Daten.

Um die Rahmenbedingungen nach der Fusion und die unternehmensspezifischen Besonderheiten adäquat zu berücksichtigen, wurden in das Projekt Vertreter aller betroffenen Teilunternehmen eingebunden. 
Die im Vorgehensmodell optionale Studie wurde durchgeführt und diente der genaueren Evaluation des Projektrisikos, der Abschätzung der Machbarkeit und dem Aufzeigen von Lösungsalternativen. Diese zusätzliche Phase wurde aufgrund der bisher geringen Erfahrungen mit konzernweiten Projekten und zur konkreteren Abschätzung der Aufwände und Kosten für notwendig erachtet.

Das Fachkonzept, das dieser Studie folgte, wurde durch das Projektteam in enger Abstimmung mit der Fachabteilung in einem iterativen Verfeinerungsprozess erstellt. Es enthält die detaillierten Beschreibungen von Schnittstellen, Datenhaltung, Datenbereitstellung, Analyse- und Selektionsmöglichkeiten sowie das Testkonzept. Im Rahmen des Fachkonzepts wurde auch festgelegt, dass das bereits bestehende Data Warehouse der Württembergischen Versicherungsgruppe die Basis für die dispositive W\&W Kundendatenbank bilden sollte.

Beim Entwurf des $D V$-Konzepts wurden die Definitionen und Erläuterungen des Fachkonzepts auf die konkret zu verwendende Informationstechnik abgebildet. Dabei wurde detailliert auf Hersteller, Produkte, Speicherbedarf, technische Verfahren und organisatorische Zuordnungen eingegangen. Das DV-Konzept enthält Beschreibungen zur systemtechnischen Architektur, d. h. zu Hard- und Software, zum Datenbank-Design sowie zum Systembetrieb.

In der Realisierungsphase erfolgte der Aufbau des Data Warehouse durch ein KernProjektteam von ca. fünf Mitarbeitern aus den IT-Abteilungen der drei Unternehmen Leonberger Bausparkasse, Wüstenrot Bausparkasse und Württembergische Versicherungsgruppe unter Leitung eines externen Projektleiters. Die Selektionen in den operativen Systemen wurden von den die jeweiligen Anwendungssysteme betreuenden Mitarbeitern in ihren gewachsenen bisherigen Zuständigkeiten erledigt, koordiniert vom Projektleiter. Getestet wurde einerseits durch die IT anhand von Testdaten, die zu vordefinierten Testfällen aus den Originalbeständen der liefernden Gesellschaften extrahiert wurden, andererseits durch die Marketing-Abteilungen der einzelnen Gesellschaften auf der Basis der ersten Übernahme von Originaldaten.

Die erste Stufe der W\&W Kundendatenbank wurde erfolgreich eingeführt. Direkt angeschlossen wurde eine erste Ausbaustufe mit dem Projekt WWK Stufe 2. Für dieses Projekt ist die organisatorische Abwicklung um vieles leichter, da inzwischen die W\&W Informatik unter einheitlicher Leitung agiert.

\subsection{Kraftfahrt-Controlling}

\subsubsection{Inhalt des Projektes}

Die Sparte Kraftfahrt stellt einen wichtigen Bestandteil im Geschäftsfelderportfolio der W\&W AG dar. Um den Chancen und Herausforderungen dieses hart umkämpften Marktes zu begegnen sowie die Ertragskraft der Sparte zu verbessern, sind ge- 
naue und zeitnahe Informationen über die Geschäftsentwicklung von entscheidender Bedeutung.

Die Informationssysteme für das Kraftfahrt-Controlling waren funktional und vor allem datenhaltungstechnisch an ihre Grenzen gelangt. Sie waren für die Unterstützung bei der Umsetzung der Spartenziele

- Optimierung der Geschäftssteuerung,

- Empfehlung von ertragsverbessernden Massnahmen und

- Unterstützung der dezentralen Strukturen

durch verbesserte Analysen zur Erkennung der Ursache-Wirkungs-Zusammenhänge nicht mehr ausreichend gerüstet.

Das neue Controlling System Kraftfahrt (CSK) soll die unterschiedlichen Inhalte und Sichtweisen der ursprünglichen Systeme integrieren und konsistente Informationen und Analysen bereitstellen. Aufgrund der bisherigen starken Autonomie der Fachabteilung hinsichtlich Entwicklung und Erweiterung der Informationssysteme besteht weiterhin der starke Wunsch nach Flexibilität und Spontaneität. Deshalb wird in diesem Projekt ein prototypisches Vorgehen verwendet, um sowohl die grundsätzliche Machbarkeit als auch erste, kurzfristige Ergebnisse aufzuzeigen. Diese sollen dann in weiteren Schritten zunehmend verfeinert und erweitert werden.

Erstes Ziel ist die Schaffung einer Basis für die Beurteilung von Schadenverläufen. Darüber hinaus sollen die Schadenbearbeitung sowie die Schadenregulierung unterstützt werden. Dazu sollen alle Schadenstammdaten, Schadenbuchungsdaten, Schadenadressen und Meldedaten in geeigneter, aufbereiteter Form in das Data Warehouse integriert werden.

In weiteren Schritten sollen auch fein granulare Schadendaten mit tagesaktueller Historie im Data Warehouse vorgehalten werden. Die Granularität soll bestandsseitig von der existierenden Policen- und Vertrags- auf Gefahrenebene verfeinert werden. Als Anwendungen sind Deckungsbeitragsrechnungen und Rentabilitätsbetrachtungen vorgesehen.

\subsubsection{Vorgehen}

Das Vorgehen im CSK-Projekt war auf das Vorgehen im WWK-Projekt abgestimmt. Es war bereits als Folgeprojekt des ersten konzernweiten Data-Warehouse-Projektes angelegt. So sollte die durch das WWK-Projekt geschaffene konzernweite Kundenbasis so um versicherungstechnische Vertrags- und Schadendaten erweitert werden, dass eine integrierte Sicht auf die Kunden möglich wird. Schon für diese beiden Projekte selbst, vor allem aber für Folgeprojekte im Data-Warehouse-Umfeld sollten sich so Synergien realisieren lassen.

Als Einzelprojekte betrachtet wurden im Kraftfahrt-Controlling-Projekt und im WWK-Projekt jeweils andere Vorgehensweisen - prototypisch gegenüber klar pha- 
senorientiert - gewählt. Die veränderte Vorgehensweise fand in den Vorgaben für die zu erstellenden Ergebnisdokumente Projektauftrag, Fachkonzept und DV-Konzept jedoch keinen Niederschlag.

Vor allem in der Diskussion mit der Fachabteilung hat sich die prototypische Vorgehensweise als äusserst positiv und zielführend herausgestellt. Schwierigkeiten bereitete allerdings der Umstand, dass es kaum mehr möglich war, zusätzliche Anforderung an den Prototypen abzulehnen. Das Argument, eine Phase oder Stufe sei abgeschlossen, war weitgehend wirkungslos.

Bei der Betrachtung der Gesamtkonzeption eines Data Warehouse innerhalb eines Unternehmens ist die prototypische Vorgehensweise global angewandt eine zwingende Folge der Strategie, das Data Warehouse über Fachprojekte und nicht über grössere Infrastrukturmassnahmen zu entwickeln.

\subsection{Risikomanagement}

\subsubsection{Inhalt des Projektes}

Als drittes Projekt wurde das Risikomanagement betrachtet. Das Konzernrisikomanagement soll zeitnah und aussagekräftig über die risikorelevanten Sachverhalte im Konzern informieren. Die Verpflichtung dafür besteht sowohl aufgrund gesetzlicher Auflagen als auch aufgrund interner Anforderungen im Hinblick auf eine risikoorientierte Konzernsteuerung. Das Gesetz zur Kontrolle und Transparenz im Unternehmensbereich (KonTraG) erweitert die Auskunftspflichten und Kontrollmöglichkeiten von Kapitalgesellschaften erheblich und soll somit zur Sicherung des Fortbestandes des Unternehmens beitragen. Alle unternehmerischen Risiken, strategische und operative, sollen rechtzeitig erkannt, überwacht und abgewehrt oder aber zumindest gemindert werden.

Das bisherige Reporting und die Datenversorgung beruhen auf Excel-Dateien, die in einem mehrstufigen, vorwiegend manuellen Prozess erstellt und verdichtet werden. Diese Vorgehensweise stellt aufgrund der grossen Anzahl von Schnittstellen und der manuellen Transformationen, mit nur teilweise dokumentierten fachlichen Berechnungsvorschriften, eine grosse Fehlerquelle, insbesondere hinsichtlich der Datenqualität und -konsistenz dar.

\subsubsection{Vorgehen}

Das Projekt befand sich zum Zeitpunkt der Erhebung noch in der Planungs- und Designphase.

Zur Erreichung einer besseren Lösung wird ein 3-Stufenplan propagiert. In der ersten Stufe soll eine multidimensionale Datenbank erstellt werden, in der alle relevanten Daten erfasst und integriert werden. Die Daten sollen entweder per ExcelSchnittstelle importiert oder per Abfragedialog erfasst werden. In einem zweiten 
Schritt wird Excel durch ein mächtigeres Auswertungstool ersetzt und in der dritten und letzten Ausbaustufe soll die Datenversorgung durch Anbindung an das Konzern-Data-Warehouse automatisiert werden.

Ein besonders kritischer Punkt ist die Überzeugung der Fachabteilung von den Vorteilen einer integrierten, konsistenten, konzernweiten Datenbasis im Vergleich zur unabhängigen, individuellen Lösung. Diskussionen ergaben sich hinsichtlich der Anforderungen der Fachabteilung an die Flexibilität der Auslösung und die Dauer des ETL-Vorgangs. Der ETL-Prozess soll praktisch jederzeit angestossen werden können und die Daten sollen daraufhin innerhalb weniger Stunden für die Analyse zur Verfügung stehen.

\subsection{Problembereiche im Vorgehen}

\subsubsection{Umfassendes Konzept durch kleine Schritte realisieren}

Ein konzernweites Data Warehouse inklusive Einbindung aller entscheidungsrelevanten Datenquellen und Versorgung sämtlicher Auswertungen ist ein Ziel, dessen Erreichung eine grosse Herausforderung darstellt und einer umfassenden Strategie bedarf. Die Data-Warehousing-Strategie muss in die IT- sowie die Unternehmensstrategie eingebettet und permanent aktualisiert werden. Die Realisierung kann nicht in einem Wurf erfolgen.

Der Leitsatz „Think big - start small“ spielt beim Data Warehousing eine wichtige Rolle. „Start small“ bedeutet, dass kleinere, abgegrenzte Teilprojekte abgeleitet und zügig umgesetzt werden müssen, so dass baldige Projektfortschritte sichtbar werden. „Think big“ sagt, dass die Umsetzung der Strategie in der mittel- bis langfristigen Planung verankert werden muss. Daher ist hierbei insbesondere darauf zu achten, dass das zu erreichende Ziel sehr klar und eindeutig formuliert wird.

\subsubsection{Wirtschaftlichkeit und Nutzen}

Bei den betrachteten Data-Warehouse-Projekten war eine Wirtschaftlichkeits- und Nutzenbetrachtung auf Basis von Erfahrungen und Schätzungen der Projektleiter festzustellen. Eine Vorlage zur strukturierten Erfassung von ökonomischen Aspekten eines Projekts unterstützte sie dabei. Da die Projekte durch gesetzliche Erfordernisse oder strategische Unternehmensziele begründet wurden, rückte die Wirtschaftlichkeitsbetrachtung eher in den Hintergrund. Eine über die Vorlagen hinausgehende methodische Unterstützung war so nicht erforderlich. Die Unterstützung durch einen Sponsor war von vorneherein gegeben.

Bei einem langfristigen, strategischen Infrastrukturprojekt wie z. B. dem Aufbau einer integrierten Datenbasis, das bereichs- und unternehmensübergreifende Veränderungsprozesse auslösen kann, ist dagegen die Gewinnung der Zustimmung und der langfristigen Unterstützung durch die Budgetverantwortlichen oft schwierig. Sie ist hier aber besonders wichtig. Oft sind auch die Kosten- und Nutzengrössen eines 
Data Warehouse verhältnismässig einfach zu identifizieren, jedoch schwer zu quantifizieren.

Als methodische Lösung bietet sich hier die Erstellung von Business Cases an. Ein Business Case hilft bei der Darstellung der Vorteile, Nutzenpotenziale, Wirtschaftlichkeitsberechnungen und Risikoabschätzungen. Er dient als Kommunikationsbasis, Dokumentation, Entscheidungsgrundlage und zum Projektmarketing. Die Erstellung von Business Cases sowohl für das Gesamtvorhaben als auch für die Teilprojekte ist daher sehr anzuraten.

\subsubsection{Datenmodellierung}

Eine weitere Besonderheit einer Data-Warehouse-Entwicklung ist die frühzeitige, möglichst vollständige und detaillierte Anforderungsdefinition und Modellierung, der Datenobjekte und -strukturen. Beim Funktionsdesign ist es vor allem - und fast ausschliesslich - wichtig die auf die Datennutzung bezogenen und benötigten Funktionen sowie die Datenextraktionen aus den Liefersystemen zu beschreiben, soweit hierfür keine Standard-Lösungen implementiert werden. Als Front-end-Tools für Auswertungen werden meist Standardprodukte eingesetzt, so dass eine detaillierte Beschreibung z. B. des Aussehens benötigter Reports nicht notwendig ist. Wichtig ist nur die für die Auswahl des Produktes entscheidungsrelevante Funktionalität.

\subsubsection{Spezifikation der Informationsbedarfe}

In den drei betrachteten Projekten gab es jeweils unterschiedliche Schwierigkeiten bei der Explizierung und Abgrenzung der geforderten Informationsbedarfe und Funktionalitäten. Während bei WWK die Hauptschwierigkeiten darin lagen, die jeweils identischen Informationen aus den unterschiedlichsten operativen Systemen zusammenzufinden, wurden bei den beiden anderen Projekten die Anforderungen der Fachabteilungen vor dem Hintergrund ihrer Kenntnis der operativen Systeme und vor allem der dort enthaltenen Datenfelder formuliert, d. h. es gab in der ersten Phase überwiegend Anforderungen, die bisher verwendeten Daten aus bestimmten Tabellen oder Spalten eines operativen Vorsystems in einem neuen Tool zur Verfügung zu stellen. Eine quellsystemunabhängige oder eine über mehrere Systeme hinweg abgeglichene Fachbegriffs- und Datendefinition zu erstellen, war bei allen drei Projekten ein zeit- und kostenintensiver Prozess.

Das Verständnis der Projektbeteiligten für Konsolidierung, Transformation und Integration in das Data Warehouse als Aktivitäten in einem Data-Warehouse-Projekt muss erst geweckt werden. Auch Designparadigmen wie die Entkopplung von operativen und analytischen Systemen erklären sich nicht von selbst. Bei Start eines Projektes sollten daher zuerst in Workshops allen Projektbeteiligten Data-Warehouse-Grundlagen vermittelt werden. Die Formulierung und Konkretisierung der fachlichen Anforderungen sollte mit strukturierten Interviews und standardisierten Dokumenten unterstützt werden. Ganz allgemein könnte die Entwicklung spezieller 
Formulare für die Dokumentation von Data-Warehouse-Projekten sehr hilfreich bei der Überwindung dieser Hürden sein.

\subsubsection{Entwicklungs- vs. Ergebnisdokumentation}

Die Projektdokumentationen werden i. A. phasenbezogen erstellt und offiziell abgenommen. Die Dokumentation erfolgt ,vorwärts orientiert“, d. h. in einer Folgephase werden Änderungen am Konzept, die sich nie ganz vermeiden lassen, nicht "rückwärts orientiert" auch in Dokumenten der Vorgängerphasen eingearbeitet. Bei Projekteinführung sind bereits einige Phasen abgearbeitet worden, der Stand der Dokumente im Fachkonzept entspricht nicht mehr dem Stand im DV-Konzept und beides stimmt nicht mit der tatsächlichen Realisierung überein. Die Dokumente einer Phase sind als Basis zum Start der nächsten Phase jeweils korrekt, darüber hinaus oft nicht. Vermieden werden könnte dies bei einem Phasenvorgehen nur, wenn bei jeder Änderung ein Rückschritt in die früheren Phasen unternommen würde. Darüber hinaus bedeutet dieses Vorgehen z. B. auch, dass von den technischen Datenelementen, den tatsächlichen Datenablagen, die Verbindungen zu den fachlichen Anforderungen oder Begriffen fehlen. Dies erschwert auch eine retrospektive Betrachtung und Qualitätsverbesserung der Projekte bzw. ihrer Ergebnisse und ihre weitergehende fachliche Nutzung.

Für eine mögliche Lösung wurde in den betrachteten Projekten eine genaue Sichtung der erforderlichen Dokumente vorgenommen. Die Teile der Phasendokumente, die in späteren Phasen und in späteren Ausbaustufen (ob in einem weiteren Projekt oder im Rahmen der normalen Wartung) aktuell gehalten werden sollten, wurden ausgelagert. Die ausgelagerten Dokumente müssen über alle Phasen und Ausbaustufen hinweg gepflegt werden. Damit der zusätzliche Aufwand erbracht werden kann, ist es notwendig, nur die wichtigsten und später auch nutzbaren Dokumente auszulagern. Auch unter diesem Gesichtpunkt sind speziell für Data-WarehouseProjekte entwickelte, dort aber standardisierte Dokumentationsformulare sehr von Nutzen.

Eine solche durchgängige Dokumentation sollte auch Informationen für den produktiven Einsatz enthalten. Nach Übergabe des implementierten Teilsystems in die Produktion wird so der Administrations- und Pflegeaufwand reduziert.

\subsubsection{Werkzeugeinsatz}

In den betrachteten Projekten wurden die Entscheidungen über den Einsatz von Tools unter der Rahmenbedingung getroffen, dass vorrangig bereits im Konzern eingesetzte Werkzeuge zum Einsatz kommen und ggf. bereits vorhandenen Lizenzen im Konzernverbund genutzt werden sollten. Eine Evaluation der eingesetzten Werkzeuge für die Nutzung in den Fachabteilungen erfolgte daher nur insoweit, als die funktionale Eignung für die jeweiligen Anforderungen der Fachabteilung gegeben sein musste. Darüberhinaus wurde nach den Wünschen der Fachabteilung ent- 
schieden. Eine Standardisierung der Schnittstellen erfolgte in allen drei Projekten nicht explizit.

Zur mittel- bis langfristigen Konsolidierung, Vereinfachung und Optimierung der Infrastruktur sollte dieses Verfahren beibehalten werden und bereits unterstützte, im Konzern üblichen Werkzeuge auch in neuen Projekten eingesetzt werden. Nur in Ausnahmefällen sollten zusätzliche Tools, für die bisher noch kein Know-how vorhanden ist, zum Einsatz kommen. Bei einer Vereinheitlichung, Reduzierung und Wiederverwendung von Datenformaten und -strukturen sollten allgemeine, zukunftssichere Standards wie z. B. XML oder CWM Einsatz finden.

\section{Data-Warehouse-Vorgehensmodell}

\subsection{Rahmenbedingungen}

Bei der Abwicklung von IT-Projekten der W\&W Informatik gelten einige Rahmenbedingungen, die Auswirkungen auf mögliche Vorgehensmodelle haben.

Für einige Phasen eines Software-Engineering-Projektes existiert ein konzernweites Vorgehensmodell, welches die zu durchlaufenden Phasen auf oberster Ebene spezifiziert und obligatorische Ergebnisdokumente der einzelnen Schritte definiert. Dieses Phasenmodell ist auch für Data-Warehouse-Projekte massgeblich. Das zu entwickelnde Data-Warehouse-Vorgehensmodell muss sich sowohl an den Phasen als auch den Ergebnisdokumenten orientieren.

Das Data-Warehouse-Vorgehensmodell soll darüber hinaus explizit die Applikationslandschaft der W\&W AG und konzernspezifische Rahmenbedingungen, wie organisatorische Regelungen und Strukturen, berücksichtigen.

Andererseits muss der mehr datenorientierten Ausrichtung der Data-WarehouseProjekte sowie der Tatsache, dass eine spätere Nutzung durch weitere Anwender zum Zeitpunkt der Projektarbeit nicht bekannt ist, Rechnung getragen werden. Zusätzlich zum herkömmlichen Vorgehen muss auch ein explizit prototypischer Entwicklungsprozess möglich sein und durch spezielle Konstrukte unterstützt werden. Verständigungsproblemen mit dem Auftraggeber sollte vorgebeugt und eine bessere Einbindung der zukünftigen Datenverwender sollte erreicht werden.

Wenn das Vorgehensmodell eine tatsächliche Hilfe in der Projektarbeit sein soll, darf der Detaillierungsgrad der Aktivitäten nicht zu gering sein. Generische Aktivitäten sind zu vermeiden. Die Operationalisierbarkeit der einzelnen Vorgänge soll stets gegeben sein. Leitfäden und Checklisten garantieren eine hohe Praktikabilität und Umsetzbarkeit des Vorgehensmodells und damit die Einsparung von Entwicklungskosten. 


\subsection{Beschreibung des Modells}

Das Data-Warehouse-Vorgehensmodell wird auf oberster Ebene als Phasenmodell bestehend aus fünf einzelnen Phasen vorgeschlagen:

Ausgehend von einer fachlichen Problemstellung wird ein umfassender Business Case erstellt, mit dem Ziel, die Ist-Situation zu analysieren, Mängel zu identifizieren, Kosten/Nutzen zu benennen und daraus unter Berücksichtigung der Rahmenbedingungen einen oder mehrere Lösungsvorschläge, ggf. in Form eines Stufenkonzepts zu erarbeiten. Aus dem Business Case resultiert der Projektauftrag.

Der Projektauftrag stellt die Grundlage für die Entwicklung des Fachkonzepts dar, das die fachlichen Aspekte detailliert. Bei der Dokumentation des Fachkonzepts wird unterschieden zwischen der tatsächlichen Phasendokumentation, die sich nur auf diese Phase und dieses Projekt bezieht und z. B. Ziele, Anforderungen, das Testkonzept und die Beschreibung der weiteren Vorgehensweise enthält, und der Dokumentation, die in späteren Phasen und Ausbaustufen fortgeschrieben werden muss, wie z. B. Datenbeschreibungen, Schlüsselwerte, usw.

Das Fachkonzept wird im Rahmen des $D V$-Konzeptentwurfs an die zu verwendende Informationstechnik angenähert. Im Rahmen des DV-Konzepts wird eine phasenund projektspezifische Dokumentation erstellt und ausserdem die im Fachkonzept erstellte separate Dokumentation um DV-technische Komponenten und grobe, fachliche Ablaufpläne ergänzt. Ebenfalls Bestandteil des DV-Konzepts sind Programmvorgaben, ggf. auf einer relativ hohen Ebene.

Die eigentliche Implementierung des Data-Warehouse-Systems findet in der Realisierungsphase statt. Auch in dieser Phase werden die in Fach- und DV-Konzept erstellten Dokumente weiter gepflegt, die Programmhandbücher werden verfeinert und als separate Dokumente abgelegt, die Ablaufpläne werden detailliert.

Die Produktivschaltung erfolgt im Rahmen der Einführung.

Dieser Phasenablauf stellt das idealtypische Vorgehen in einem Entwicklungsprojekt dar. Grundsätzlich jedoch wird nach jeder der fachlich orientierten Phasen ein Managemententscheid über die Fortsetzung bzw. den Abbruch des Projekts eingeholt. Das Phasenmodell des Data-Warehouse-Vorgehens auf der obersten Ebene zeigt Abb. 2.

Als zusätzliche Aktivität im Vorgehen kann parallel zu den ersten drei Phasen optional eine Projektstudie initiiert werden. Diese stellt ein eigenständiges Projekt dar mit dem Ziel, eine Expertise zu einem bestimmten Themenkomplex oder einem der Lösungsvorschläge zu erarbeiten.

Das hier dargestellte Phasenmodell sieht auch ein prototypisches Vorgehen nach der Erstellung des Business Case vor. Prototypen sind z. B. in Situationen sinnvoll, in denen 


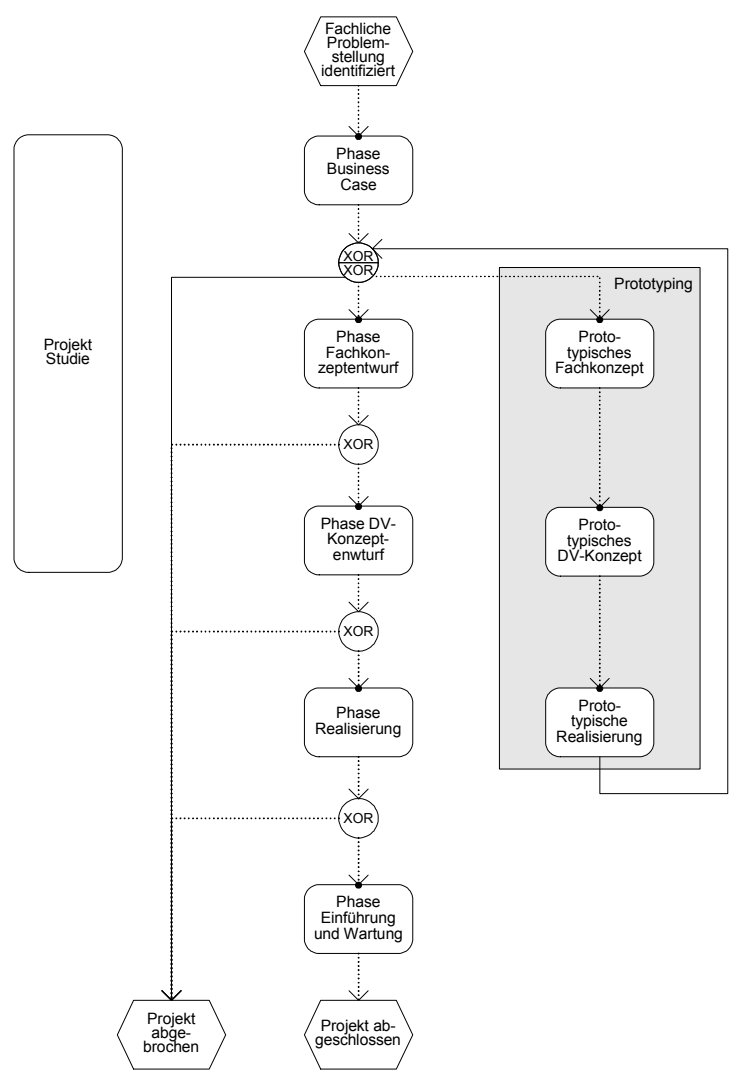

Abb. 2: Phasen des Data-Warehouse-Vorgehensmodells

- die Endbenutzer ihre Anforderungen nicht hinreichend genau explizieren können,

- ein frühzeitig funktionsfähiges, aber nicht vollständiges System gefordert wird oder

- eine Machbarkeitsstudie die Unsicherheiten auf Seiten der Entwickler oder Endbenutzer ausräumen soll.

Beim Prototyping werden, in Anlehnung an das ,normale“ Vorgehen, ein Fachkonzept, ein DV-Konzept und eine Realisierung durchgeführt. Dabei stellen die jeweils auszuführenden Aktivitäten eine Teilmenge der Aktivitäten dar, die beim herkömmlichen Vorgehen durchlaufen werden. Diese Teilmenge ist abhängig von der Zielsetzung des Prototyps. Es werden Wegwerf-, Demonstrations-, Präsentations- und wiederverwendbare Prototypen unterschieden. Je nach Typ sind unterschiedliche Aktivitäten durchzuführen bzw. zu überspringen. Durch die Berücksichtigung eines prototypischen Entwicklungsprozesses ist eine effizientere und einfachere Kommu- 
nikation mit dem Auftraggeber möglich, insbesondere kann ein Prototyp bei der Explikation der Anforderungen an ein Data-Warehouse-System sehr hilfreich sein.

$\mathrm{Zu}$ jeder dieser beschriebenen Phasen existieren detaillierte Prozessmodelle, die die durchzuführenden Aktivitäten auf einer sehr feinen Granularitätsebene beschreiben und deren Abarbeitungsreihenfolge festlegen. Als Modellierungssprache wurden Ereignisgesteuerte Prozessketten ${ }^{2}$ (EPK) gewählt, da diese trotz eines angemessenen Formalisierungsgrades leicht verständlich sind und durch Modellierungswerkzeuge unterstützt werden. Die Modelle enthalten auch relevante Dokumente, die entweder Ergebnis einer Aktivität sind oder als Input für eine bestimmte Aufgabe dienen. Des Weiteren werden allen Aktivitäten konzernspezifische Rollen über Beziehungen zugeordnet. Diese Beziehungen drücken den funktionellen Zusammenhang der Rolle zu der jeweiligen Aktivität aus. Durch dieses Rollen-Modell werden u. a. die Verantwortlichkeiten klar geregelt. In Data-Warehouse-Projekten haben sich für die W\&W Informatik folgende Beziehungstypen als relevant herausgestellt:

- führt aus,

- ist fachlich verantwortlich für,

- ist dv-verantwortlich für,

- wirkt mit bei,

- muss informiert werden über,

- entscheidet über und

- bringt Interessen ein.

\section{Detailliertes Prozessmodell der Phase Business Case}

Da auf eine ausführliche Beschreibung aller Phasen und ihrer Aktivitäten aus Platzgründen verzichtet werden muss, wird die Detaillierung nur beispielhaft an der Phase Business Case erläutert. Die Abbildungen 3 und 4 zeigen das gesamte Prozessmodell der Phase Business Case. Aus Gründen der Übersichtlichkeit werden alle Aktivitäten, aber nur die für den Prozessablauf relevanten Ereignisse angegeben. Auf die Darstellung von Input- bzw. Output-Dokumenten wird ebenfalls verzichtet.

Oberstes Ziel der Phase Business Case ist es, die Zustimmung und Unterstützung des Budgetverantwortlichens bzw. Sponsors zu dem Projekt zu erlangen. Ein Business Case sollte sowohl bei der initialen Durchführung eines Data-Warehouse-Projekts als auch bei nachfolgenden Ausbaustufen aufgestellt werden. Der Business Case dient als Kommunikationsbasis sowie als Planungs- und Entscheidungsbasis für alle Projektbeteiligten.

2 Für eine detaillierte Beschreibung der Ereignisgesteuerten Prozessketten vgl. (Rosemann 2000, S. 59 ff.) und (Scheer 2001, S. 125 ff.). 


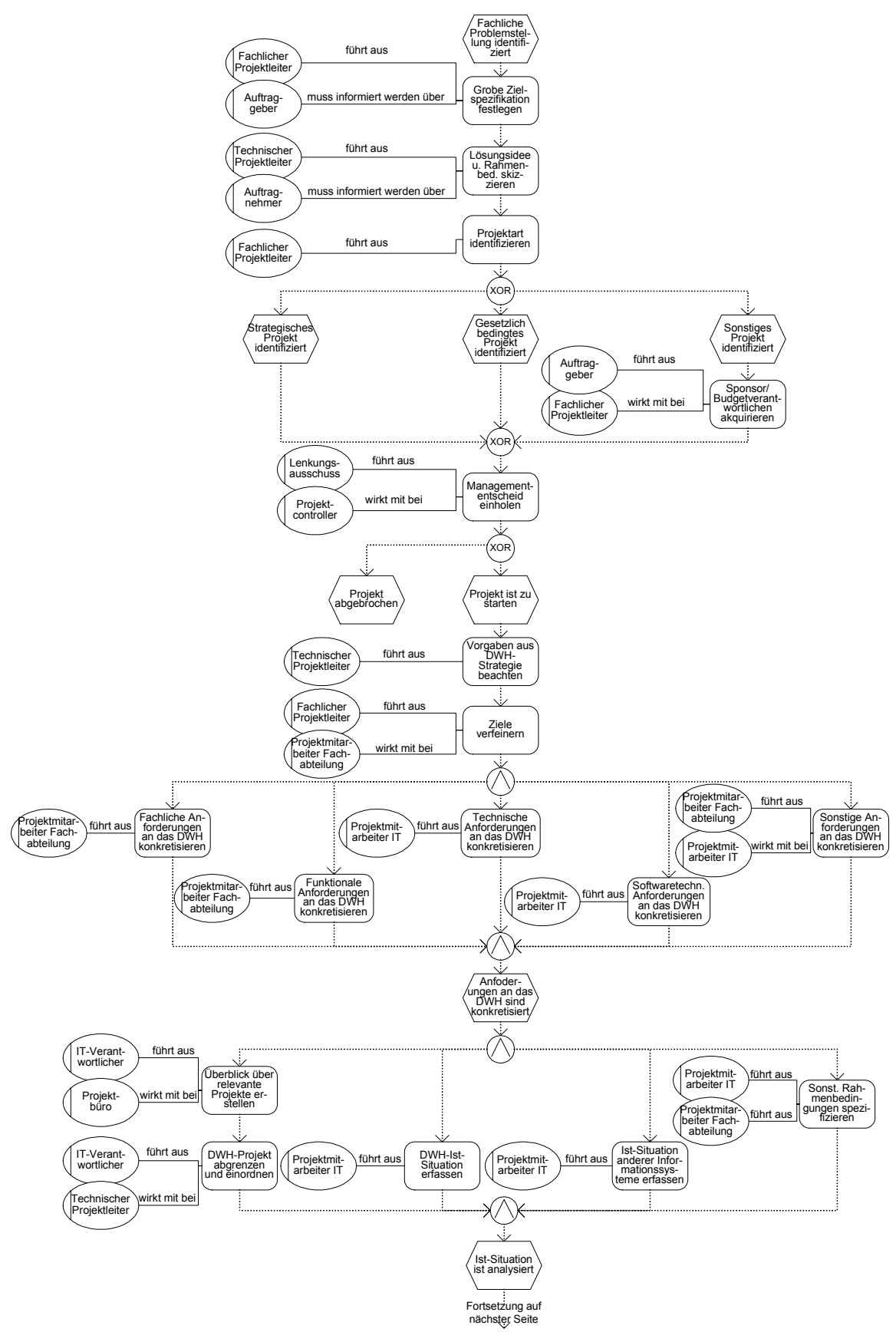

Abb. 3: Prozessmodell „Business Case“ (Teil 1) 


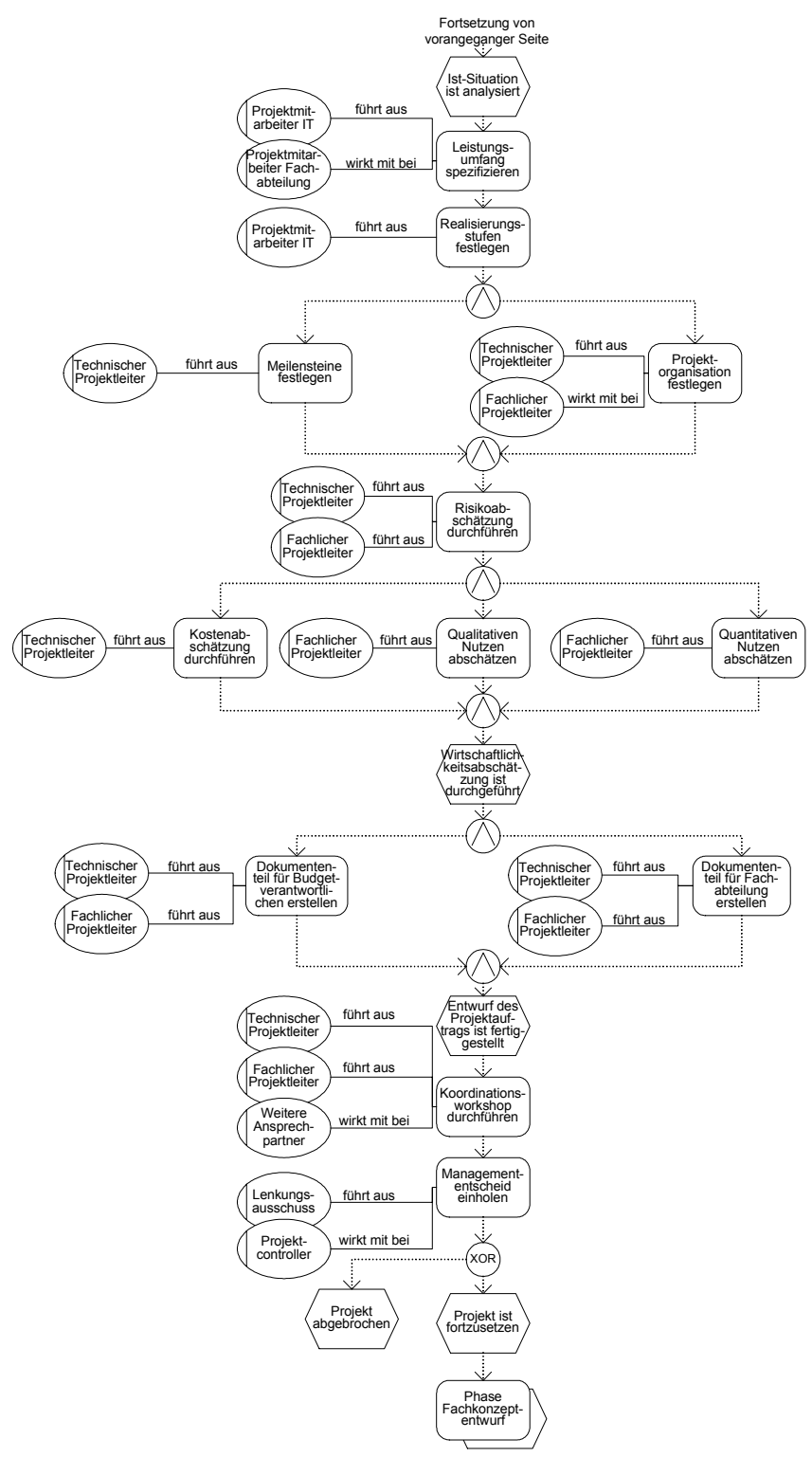

Abb. 4: Prozessmodell „Business Case“ (Teil 2)

Erster Schritt dieser Phase ist die Formulierung einer Projektanfrage durch den fachlichen Projektleiter, welche grob die Probleme und Ziele des Projekts spezifiziert. Der technische Projektleiter offeriert daraufhin eine Lösungsidee mit den zugrunde liegenden Rahmenbedingungen. Es lassen sich grundsätzlich verschiedene Auslöser für ein Projekt unterscheiden. Ist ein Projekt gesetzlich oder strategisch bedingt, tre- 
ten die Finanzierungsfragen in den Hintergrund, da diese umgesetzt werden müssen, um den Unternehmensfortbestand zu sichern. Die verantwortlichen Sponsoren sind in einem solchen Fall durch die Rahmenbedingungen determiniert. Sonstige Vorhaben jedoch werden aus Sicht der Budgetverantwortlichen als Kann-Projekte eingestuft. Deshalb müssen diese von der Wichtigkeit und dem Nutzen des Projektes durch den Auftraggeber und den fachlichen Projektleiter überzeugt werden. Anschliessend wird auf Basis der grob skizzierten Problemstellung und der dazugehörigen Lösungsidee über den Projektfortgang entschieden.

Wird das Projekt fortgesetzt, so werden als nächstes die Projektziele aus der aktuellen Data-Warehouse-Strategie abgeleitet und detailliert. Die noch sehr groben Anforderungen des Projektangebots werden im Folgenden fachlich, funktional, softwarespezifisch und technisch konkretisiert. Nach einer sorgfältigen Analyse der IstSituation werden der konkrete Leistungsumfang und die Realisierungsstufen festgelegt. Innerhalb einer Realisierungsstufe werden Meilensteine und organisatorische Rahmenbedingungen definiert.

Im Anschluss daran werden Risiken sowie Kosten und Nutzen des Entwicklungsprojekts abgeschätzt und bewertet. Gerade diese Aktivitäten weisen bei der Data Warehouse-Entwicklung Besonderheiten auf, die für herkömmliche Software-Engineering-Projekte nicht zutreffend sind. Folgende Risiken sind typisch für DataWarehouse-Entwicklungsprojekte:

- Geringe Relevanz der Daten im Data Warehouse für die Datenverwender,

- unzureichende Datenqualität,

- langfristig Akzeptanz- und Vertrauensverlust in das Data-Warehouse-System,

- kein abgeschlossenes Projekt sondern ständig neue, ausufernde Anforderungen der Nutzer an das Data Warehouse und

- hohes Konfliktpotenzial aufgrund einer Vielzahl unterschiedlichster Anspruchsgruppen im gesamten Unternehmen.

Neben den Risiken stellen die Nutzenpotenziale eines Data Warehouse eine weitere Eigenart von derartigen Entwicklungsprojekten dar. Hierbei überwiegen qualitative, nicht messbare Aspekte im Gegensatz zu den meist quantifizierbaren Nutzengrössen bei der Entwicklung herkömmlicher Softwaresysteme:

- Aktuellere Daten und schnellere Informationsverteilung und-versorgung,

- effiziente und zeitnahe Entscheidungen,

- qualitativ hochwertigere Daten im Data Warehouse und den operativen Systemen,

- Realisierung einer unternehmensweiten Sicht und Analyse,

- Standardisierung von Begriffen und Kennzahlen, 
- Vermeidung von Redundanzen und Inkonsistenzen,

- Dezentralisierung von Entscheidungen und

- kürzere Suchzeiten.

Nach einer eingehenden Risiko- und Wirtschaftlichkeitsanalyse wird der Projektauftrag erstellt, der das Hauptergebnis dieser Phase darstellt und auf die beiden Zielgruppen Budgetverantwortlicher und Fachabteilung auszurichten ist. Bestimmte Teile des Projektauftrags sind jeweils auf eine der beiden Zielgruppen fokussiert. Schwerpunkte für den budgetverantwortlichen Entscheidungsträger sind die Nutzenaspekte und die Wirtschaftlichkeitsbetrachtungen. Er muss davon überzeugt werden, dass das Projekt ein Gewinn für ihn und das Unternehmen ist. Die Fachabteilung dagegen fokussiert sich auf fachspezifische Aspekte. Die zusammengetragenen Ergebnisse werden in Form eines Entwurfs des Projektauftrags in einem gemeinsamen Workshop von Fach- und IT-Abteilung diskutiert und abgestimmt. Auf Basis des abgestimmten Projektauftrags wird ein Managemententscheid über den zukünftigen Projektverlauf herbeigeführt. Hiermit endet die Phase Business Case und leitet über in die nächste Phase, nämlich den Fachkonzeptentwurf.

\subsection{Erreichte Verbesserungen beim Vorgehen}

Grundsätzlich können die Prozessmodelle und deren Beschreibungen als dokumentiertes, organisationales Wissen verstanden werden, welches nun unabhängig von Personen jederzeit allen Mitarbeitern zur Verfügung gestellt und so als Teil des organisationalen Wissensmanagement angesehen werden kann. Das Data-WarehouseVorgehensmodell selbst berücksichtigt die Anforderungen der W\&W Informatik und versucht, den identifizierten Schwierigkeiten bei den bisherigen Projekten (vgl. Kapitel 3.4) so weit wie möglich entgegenzuwirken.

Durch die explizite Berücksichtigung eines prototypischen Vorgehens wird versucht, erste für die Fachabteilung nutzbare Ergebnisse möglichst schnell zu realisieren und so die Akzeptanz zu steigern und Überzeugungsarbeit hinsichtlich einer Data-Warehouse-Lösung zu leisten. Auch können durch eine prototypische Realisierung die fachlichen Erfordernisse im Rahmen der Anforderungs- und Informationsbedarfsanalyse schneller und umfassender erhoben werden.

Eine weitere wichtige Veränderung stellt die Einführung eines Business Case dar. Dieser bindet bereits vor dem eigentlichen Projektstart die Sponsoren, Budgetverantwortlichen, Nutzer und Entwickler in das Projekt ein und schafft so eine gemeinsame Basis für die Zusammenarbeit. Durch eine zielgruppenspezifische Ausrichtung des Business Case werden alle Projektbeteiligten adäquat angesprochen, indem ihre jeweiligen Informationsbedürfnisse optimal befriedigt werden. Hierdurch lässt sich insbesondere auch die langfristige Unterstützung des Projekts sicherstellen.

Das ausgefeilte Rollenkonzept, welches jeder Aktivität eine oder mehrere Rollen zuordnet, legt Verantwortlichkeiten, Aufgabenbereiche und einzubeziehende Perso- 
nen genau fest. Hierdurch wird vor allem die Kommunikation und Abstimmung zwischen den Projektbeteiligten erleichtert und Konflikten wird bereits im Vorfeld entgegengewirkt.

Das Vorgehensmodell spezifiziert Dokumente und ordnet diese den Aktivitäten als Input oder Output zu. Eine Auflistung wichtiger Dokumente liefert Tabelle 1. Es wird ersichtlich in welcher Phase welches Dokument erzeugt und genutzt wird. Derartige Übersichten und Checklisten lassen sich in einfacher Weise aus den Prozessmodellen ableiten und können so als Hilfsmittel bei der Durchführung von DataWarehouse-Projekten eingesetzt werden. Da das gesamte Vorgehensmodell mittels eines Modellierungswerkzeugs erstellt wurde, sind diese Checklisten automatisch oder semi-automatisch generierbar.

Zur Sicherstellung einer konsistenten Dokumentation wird zwischen Entwicklungsund Ergebnisdokumenten unterschieden. Letztere stellen die Teilmenge der Entwicklungsdokumentation dar, die für zukünftige Tätigkeiten, wie bspw. die Wartung oder die Weiterentwicklung, notwendig erscheinen. Hierdurch wird einerseits der Dokumentations- und Pflegeaufwand auf ein Minimum reduziert und andererseits wird eine ausreichende, aktuelle Dokumentation der Systeme bereitgestellt, die langwierige Einarbeitungsprozesse vermeiden soll.

Ein Beispiel hierfür sind im Rahmen der Data-Warehouse-Entwicklung die Beschreibungen der im Kern-Data-Warehouse enthaltenen Daten. Diese stellen sowohl im Fach- als auch im DV-Konzept einen zentralen Bestandteil dar und müssen auch nach Abschluss der jeweiligen Phase in Form einer Ergebnisdokumentation weitergepflegt werden, da auch nach den Entwicklungsphasen ständig Änderungen und Anpassungen der Datenbasis des Data-Warehouse-Systems notwendig sind. Im Fachkonzept werden die Inhalte des Data Warehouse aus fachlicher Sicht spezifiziert. Hierzu werden die notwendigen Informationsobjekte und dazugehörige Eigenschaften (identifizierende und beschreibende) definiert, die die zentralen Entitäten des Data Warehouse bilden. Es erfolgt ausschliesslich eine fachliche Beschreibung ohne Berücksichtigung technischer Aspekte. Ausgehend von dieser Spezifikation werden den fachlichen Datenbeschreibungen im DV-Konzept techniknahe Datenspezifikationen zugeordnet. Unter anderem werden Datentypen, Datenformate, Wertebereiche, Tabellennamen und Schlüssel festgelegt. Die obigen Informationen sind zentral für die Entwicklung eines Data Warehouse und bleiben auch nach Fertigstellung einer ersten Ausbaustufe von entscheidender Bedeutung. Daher müssen diese Teile des Fach- bzw. DV-Konzepts kontinuierlich gepflegt und aktuell gehalten werden. 


\begin{tabular}{|c|c|c|c|c|c|c|}
\hline \multirow[b]{2}{*}{ Dokument } & \multirow[b]{2}{*}{ Kurzbeschreibung } & \multicolumn{5}{|c|}{ Phasen } \\
\hline & & 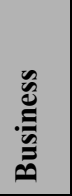 & 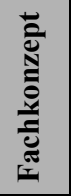 & 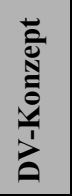 & 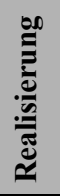 & 些 \\
\hline Projektanfrage & $\begin{array}{l}\text { Anforderungen der Fachabteilung für ein } \\
\text { zukünftiges Projekt }\end{array}$ & $\mathrm{O}, \mathrm{I}$ & & & & \\
\hline Projektangebot & $\begin{array}{l}\text { Erste Lösungsidee der IT-Abteilung bzgl. } \\
\text { der Projektanfrage (nicht detailliert) }\end{array}$ & $\mathrm{O}, \mathrm{I}$ & & & & \\
\hline $\begin{array}{l}\text { Template Risi- } \\
\text { kobeurteilung }\end{array}$ & $\begin{array}{l}\text { Extern vorgegebene Vorlage zur Bestim- } \\
\text { mung des Risikos eines Projekts anhand } \\
\text { zahlreicher Kriterien }\end{array}$ & I & & & & \\
\hline $\begin{array}{l}\text { Template Wirt- } \\
\text { schaftlichkeits- } \\
\text { beurteilung }\end{array}$ & $\begin{array}{l}\text { Extern vorgegebene Vorlage zur Bestim- } \\
\text { mung der Wirtschaftlichkeit eines Pro- } \\
\text { jekts anhand zahlreicher Kriterien }\end{array}$ & I & & & & \\
\hline Projektauftrag & $\begin{array}{l}\text { Lösungsvorschlag auf konzeptionellem } \\
\text { Level; Basis für den Projektentscheid }\end{array}$ & $\mathrm{O}$ & I & & & \\
\hline $\begin{array}{l}\text { Soll-DWH- } \\
\text { Datenmodell }\end{array}$ & $\begin{array}{l}\text { Anzustrebendes Datenmodell des Kern- } \\
\text { Data-Warehouse }\end{array}$ & & $\mathrm{O}, \mathrm{I}$ & & & \\
\hline $\begin{array}{l}\text { Ist-DWH- } \\
\text { Datenmodell }\end{array}$ & $\begin{array}{l}\text { Aktuell vorliegendes Datenmodell des } \\
\text { Kern-Data-Warehouse }\end{array}$ & & I & & & \\
\hline Fachkonzept & $\begin{array}{l}\text { Detaillierter Lösungsvorschlag und Vor- } \\
\text { gehen auf fachkonzeptionellem Level }\end{array}$ & & $\mathrm{O}$ & I & & \\
\hline $\begin{array}{l}\text { Externe Marktü- } \\
\text { bersicht }\end{array}$ & $\begin{array}{l}\text { State-of-the-art-Überblick über am Markt } \\
\text { verfügbare Tools mit kritischer Beurtei- } \\
\text { lung }\end{array}$ & & & I & & \\
\hline DV-Konzept & $\begin{array}{l}\text { DV-spezifische Umsetzungen und Erwei- } \\
\text { terungen des Fachkonzepts }\end{array}$ & & & $\mathrm{O}, \mathrm{I}$ & I & I \\
\hline
\end{tabular}

Tab. 1: Dokumentenübersicht und phasenbezogene Input-/Output-Beziehung O: Output, I: Input

\section{Zusammenfassung und Ausblick}

Der vorliegende Artikel zeigt das Vorgehen zur Entwicklung eines für Data-Warehouse-Projekte spezifischen Vorgehensmodells bei der W\&W Informatik auf und beschreibt das so entwickelte Data-Warehouse-Vorgehensmodell. 
Ausgehend von drei Entwicklungsprojekten wurden Probleme und Schwierigkeiten im jeweiligen Vorgehen festgestellt und analysiert. Hinzu kamen weitere Anforderungen und relevante Rahmenbedingungen des neu entstandenen W\&W-Konzerns, die aufgenommen und berücksichtigt werden mussten. Auf dieser Basis wurde ein idealtypisches Data-Warehouse-Vorgehensmodell entwickelt. Es werden insbesondere detaillierte Aktivitäten sowie konzernspezifische Rollen und Ergebnisdokumente beschrieben. Nach unserer Überzeugung werden dabei sowohl die spezifizierten Anforderungen berücksichtigt als auch die identifizierten Probleme angegangen. Der Praxistest allerdings, der Einsatz des Vorgehensmodells in einem konkreten Projekt, steht noch aus.

Das Data-Warehouse-Vorgehensmodell soll künftig in konzernweiten Data-Warehouse-Projekten als Leitfaden und Hilfsmittel für einen einheitlichen, strukturierten und spezifischen Data-Warehouse-Projektablauf dienen.

Offen sind Änderungen und Erweiterungen, die sich aus der Validierung des erarbeiteten Data-Warehouse-Vorgehensmodells im praktischen Einsatz ggf. ergeben werden. Des Weiteren kann das Data-Warehouse-Vorgehensmodell in zukünftigen Ausbaustufen erweitert werden durch die Zuordnung von Werkzeugen bzw. Informationssystemen zu den Aktivitäten, durch Berücksichtigung von Datenschutzaspekten bspw. durch Kennzeichnung besonders schützenswerter, personenbezogener Daten und durch die Festlegung von Verantwortlichkeiten für anwendungsspezifische und anwendungsübergreifende Daten. Weiterhin können Projektmanagementaspekte sowie detaillierte Techniken noch ergänzt werden, wie bspw. Heuristiken zur Verteilung der Entwicklungskosten eines Data Warehouse auf neu hinzukommende Nutzer nach der Implementierung.

\section{Literatur}

Balzert, H.: Lehrbuch der Software-Technik: Software-Management, Software-Qualitätssicherung, Unternehmensmodellierung; Spektrum, Heidelberg und Berlin 1998.

Bremer, G.: Genealogie von Entwicklungsschemata; in Kneuper, R., Müller-Luschnat, G., Oberweis, A. (Hrsg.): Vorgehensmodelle für die betriebliche Anwendungsentwicklung, Teubner-Verlag, Stuttgart, Leipzig, 1998, S. 32-59.

Hansen, W.-R.: Vorgehensmodell zur Entwicklung einer Data Warehouse-Lösung; in Mucksch, H., Behme, W. (Hrsg.): Das Data Warehouse-Konzept: Architektur - Datenmodelle - Anwendungen, Gabler-Verlag, Wiesbaden 1997, S. 311-328.

Heuer, A., Saake, G.: Datenbanken: Konzepte und Sprachen; mitp-Verlag, Bonn 2000.

Holthuis, J.: Der Aufbau von Data Warehouse-Systemen: Konzeption - Datenmodellierung Vorgehen; Gabler-Verlag, Wiesbaden 1999. 
Jung, R., Winter, R.: Data Warehousing: Nutzungsaspekte, Referenzarchitektur und Vorgehensmodell; in Jung, R., Winter, R. (Hrsg.): Data Warehousing Strategie: Erfahrungen, Methoden, Visionen; Springer-Verlag, Berlin u. a. 2000, S. 3-20.

Kachur, R.: Data Warehouse Management Handbook, Prentice Hall, Paramus, NJ 2000.

Meyer, M., Strauch, B.: Organisationskonzepte im Data Warehousing; in Jung, R., Winter, R. (Hrsg.): Data Warehousing Strategie: Erfahrungen, Methoden, Visionen; SpringerVerlag, Berlin u. a. 2000, S. 79-100.

o. V.: Gabler Wirtschaftsinformatik-Lexikon; Gabler, Wiesbaden 1997.

Poe, V., Klauer, P., Brobst, S.: Building a Data Warehouse for Decision Support; Prentice Hall, Upper Saddle River, NJ 1997.

Rosemann, M.: Vorbereitung der Prozessmodellierung; in Becker, J., Kugeler, M., Rosemann, M. (Hrsg.): Prozessmanagement - Ein Leitfaden zur prozessorientierten Organisationsgestaltung; Springer-Verlag, Berlin u. a. 2000, S. 45-90.

Scheer, A.-W.: ARIS - Modellierungsmethoden, Metamodelle, Anwendungen; 4. Aufl., Springer-Verlag, Berlin u. a. 2001.

Simon, A.: 90 Days to the Data Mart; John Wiley \& Sons, New York u. a. 1998.

Vossen, G.: Datenmodell, Datenbanksprachen und Datenbank-Management-Systeme; Addison-Wesley, Bonn u. a. 1994. 